CORRIGENDUM

doi:10.1038/nature13676

\title{
Corrigendum: Systems survey of endocytosis by multiparametric image analysis
}

Claudio Collinet, Martin Stöter, Charles R. Bradshaw, Nikolay Samusik, Jochen C. Rink, Denise Kenski,

Bianca Habermann, Frank Buchholz, Robert Henschel, Matthias S. Mueller, Wolfgang E. Nagel, Eugenio Fava,

Yannis Kalaidzidis \& Marino Zerial

Nature 464, 243-249 (2010); doi:10.1038/nature08779

Readers alerted us to a technical mistake (a shift of one row) made during the compilation of Supplementary Table 4, resulting in the assignment of 455 gene symbols to the wrong gene IDs. For the affected genes, the cluster group, the number of positive small interfering and endoribonuclease-prepared RNAs (si/esiRNAs) per gene and the Phenoscore values were assigned to the wrong gene symbol (although they were all assigned to the correct gene ID). All data in the online database were correct. The Supplementary Information of this Corrigendum contains the corrected Supplementary Table 4. We have also redesigned our online database (containing all images and profiles) to provide a more user-friendly interface, a visual representation of the phenotypic profiles, a facilitated gene search and integration with other public internet databases. The appropriate link on page 245 of the Article should therefore be http://endosomics.mpi-cbg.de.

Supplementary Information is available in the online version of this Corrigendum. 